\title{
Metabelian groups with the same finite quotients
}

\section{P.F. Pickel}

\begin{abstract}
Let $F(G)$ denote the set of isomorphism classes of finite quotients of the group $G$. Two groups $G$ and $H$ are said to have the same finite quotients if $F(G)=F(H)$. We construct infinitely many nonisomorphic finitely presented metabelian groups with the same finite quotients, using modules over a suitably chosen ring. These groups also give an example of infinitely many nonisomorphic split extensions of a fixed finitely presented metabelian group by a fixed finite abelian group, all having the same finite quotients.
\end{abstract}

Let $F(G)$ denote the set of isomorphism classes of finite quotients of the group $G$. We say groups $G$ and $H$ have the same finite quotients if $F(G)=F(H)$. Many examples have been given of nonisomorphic groups with the same finite quotients ([11], [5], [4], [9], [12]). In each of these examples the groups are polycyclic and the number of nonisomorphic groups with the same finite quotients is finite. In fact, it has been shown ([10]) that for the class of nilpotent-by-finite groups, the number of isomorphism classes of groups with the same finite quotients must always be finite. In this paper, we construct infinitely many nonisomorphic finitely presented metabelian groups with the same finite quotients. Since metabelian groups are residually finite ([7]) and satisfy the maximal condition for normal subgroups ([6]), it seems that rather stringent conditions must hold in order that the number of groups with the same finite quotients be finite.

Received 8 April 1974. The author gratefully acknowledges the support of the National Science Foundation of the USA. 
If $R$ is a commutative noetherian ring and $M$ is an $R$-module, let $F_{R}(M)$ denote the set of $R$-isomorphism classes of finite $R$-module quotients of $M$. Two $R$-modules $M$ and $N$ have isomorphic finite quotients as $R$-modules $\left(I F Q_{R}\right)$ if $F_{R}(M)=F_{R}(N)$.

THEOREM. Let $R$ be as above and let $M$ and $N$ be finitely generated R-modules. Then $M$ and $N$ have $I F Q_{R}$ if and only if $M / \mathrm{m}^{n} M$ is isomorphic to $N / \mathrm{m}^{n} N$ for all integers $n$ and all maximal ideals $m$ of finite index in $R$.

Proof. Suppose first that $M$ is a finite $R$-module. Then the annihilator of $M, \operatorname{ann}(M)=\{r \in R \mid r m=0 \forall m \in M\}$ is an ideal of finite index in $R$ since it is the kernel of a homomorphism into the finite ring end $(M) \cdot \operatorname{ann}(M)=\mathrm{a}_{1} \cap \ldots n \mathrm{a}_{n}$, where $\mathrm{a}_{i}$ are primary ideals belonging to distinct prime ideals $p_{i}[13, p .209]$. Since $\mathrm{p}_{i} \supseteq \mathrm{a}_{i} \supseteq \operatorname{ann}(M), \mathrm{p}_{i}$ must be of finite index in $R$ and thus must be maximal, since the quotient $R / p_{i}$, being a finite integral domain, must be a field. Since $\mathrm{a}_{i} \supseteq \mathrm{p}_{i}^{k(i)}[13, \mathrm{p} .200]$, ann $(M)$ contains $\prod_{i=1}^{n} \mathrm{p}_{i}^{k(i)}$.

Now let $M$ be an arbitrary finitely generated $R$-module. Then

$$
M /\left(\prod_{i=1}^{n} \mathrm{p}_{i}^{k(i)}\right) M \cong \bigoplus_{i=1}^{n} M / \mathrm{p}_{i}^{k(i)} M,
$$

since the $\mathrm{p}_{i}$ are pairwise comaximal [13, p. 178]. If $M / \mathrm{p}^{k} M=N / \mathrm{p}^{k}$ for all integers $k$ and all maximal ideals $p$ of finite index, then by the above:

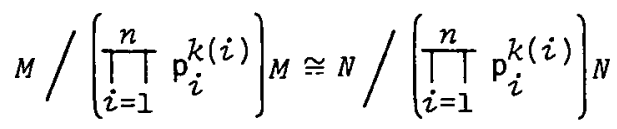

for all integers $k(i)$ and maximal ideals $\mathbf{p}_{i}$ of finite index. If $M / M^{\prime}$ is any finite quotient of $M, \operatorname{ann}\left(M / M^{\prime}\right) \supseteq \prod_{i=1}^{n} \mathrm{p}_{i}^{k(i)}$ for some integers $k(i)$ and maximal ideals $p_{i}$ of finite index (by the first paragraph). 
Since $M / M^{\prime}$ is a quotient of $M /\left(\prod_{i=1}^{n} \mathrm{p}_{i}^{k(i)}\right) M$ and (*) holds, $M / M^{\prime}$ must be isomorphic to a finite quotient of $N$. Thus $M$ and $N$ have $I F Q_{R}$.

Conversely, $M / \mathrm{p}^{k} M$ and $N / \mathrm{p}^{k} N$ are the largest quotients of $M$ and $N$ respectively with annihilator $\mathrm{p}^{k}$. If $M$ and $N$ have $I F Q_{R}$, then $M / \mathrm{p}^{k} M$ and $N / \mathrm{p}^{k} N$ must be isomorphic.

Suppose now that $A$ is a subgroup (necessarily abelian) of the group of units $U(R)$ of $R$, which generates $R$ as a ring. If $M$ is a finitely generated $R$-module, we may form the semidirect product, $M \rtimes A$, a metabelian group.

LEMMA. Let $A$ be a subgroup of $U(R)$ as above and let $M$ and $N$ be finitely generated R-modules with $I F Q_{R}$. Then the groups $M \times A$ and $N \times A$ have the same finite quotients.

Proof. Suppose $M^{\prime}$ is a normal subgroup of $M \rtimes A$ of finite index. Since $A$ generates $R$ as a ring and $M^{\prime}$ is normal, $M \cap M^{\prime}=M^{\prime \prime}$ is an $R$-submodule of $M$ of finite index. Since $M$ and $N$ have $I F Q_{R}$, there is an $R$-submodule $N^{\prime \prime}$ of $N$ such that $M / M^{\prime \prime}$ and $N / N^{\prime \prime}$ are isomorphic as $R$-modules. Thus $\left(M / M^{\prime \prime}\right) \rtimes A$ and $\left(N / N^{\prime \prime}\right) \rtimes A$ are isomorphic groups. Since $(M \times A) / M^{\prime}$ is a quotient of $\left(M / M^{\prime \prime}\right) \times A$, there must be a quotient of $N \times A$ isomorphic to $(M \times A) / M^{\prime}$.

Let $R$ be a commutative noetherian ring. A finitely generated $R$-module $M$ is a rank one projective module if and only if the localization $M_{\mathrm{p}}$ of $M$ at each prime $\mathrm{p}$ of $R$ is isomorphic to the localization $R_{\mathrm{p}}$ of $R$. Since

$$
M / \mathrm{p}^{n} M \cong M_{\mathrm{p}} / \mathrm{p}^{n} M_{\mathrm{p}} \cong R_{\mathrm{p}} / \mathrm{p}^{n} R_{\mathrm{p}} \cong R / \mathrm{p}^{n}
$$

for all positive integers $n$ and all prime ideals $p$ of $R$, all rank one projective modules over $R$ have $I F Q_{R}$. Since $M_{\mathrm{p}}=R_{\mathrm{p}}$ for each prime p, $M$ is a faithful $R$-module (that is, $r m=0 \forall m \in M$ implies $r=0$ ). The Picard group Pic $(R)$ of $R$ is the group of isomorphism classes of rank one projective modules over $R$, under tensor product. 
Let $\Pi$ be a finite abelian group not of square-free order. Let $2 \Pi$ denote the integral group ring of $\pi$ and let $x$ be an indeterminate. Then $\operatorname{Pic}(Z \Pi[x])$ is infinite [2, Theorem 8.10] and the map from $\operatorname{Pic}(2 \pi[x])$ into $\operatorname{Pic}\left(2 \pi\left[x, x^{-1},(x+1)^{-1}\right]\right)$ is injective [8, Theorem 1.3], so the latter group is also infinite. Let $R$ be the ring $2 \Pi\left[x, x^{-1},(x+1)^{-1}\right]$ and let $A$ be the abelian subgroup of the group of units of $R$ generated by $\Pi, x$ and $(x+1)$. For any rank one projective module $M$, we form the group $G_{M}=M \rtimes A$. Since $A$ generates $R$ as a ring, all the groups $G_{M}$ have the same finite quotients. Since $m^{x}=m^{(x+1)-1}=\left[m, m^{x+1}\right]$ for all $m$ in $M, M$ is contained in the derived group of $G_{M}$. Since $G_{M} / M$ is abelian, $M$ must equal the derived group $G_{M}^{\prime}$ of $G_{M}$.

PROPOSITION. The groups $G_{M}$ described above lie in infinitely many distinet isomorphism classes.

Proof. If $\psi$ is an automorphism of $R$ and $N$ is an $R$-module, we may form the twisted module $\psi_{N}$. Scalar multiplication $r * n$ in $\psi_{N}$ is defined by $r * n=\psi(r) \cdot n$ for $r$ in $R$ and $n$ in . . Two $R$-modules $M$ and $N$ are isomorphic if and only if $\psi_{M}$ and $\psi_{N}$ are isomorphic. Now let $\varphi: G_{M} \rightarrow G_{N}$ be an isomorphism. Since $M=G_{M}^{\prime}$ and $N=G_{N}^{\prime}$, $\varphi(M)=N$ and $\varphi$ induces an automorphism $\bar{\varphi}$ of $A$.

$$
\varphi(m)^{\varphi(x+1)}=\varphi\left(m^{x+1}\right)=\varphi\left(m^{x} \cdot m\right)=\varphi(m)^{\varphi(x)} \cdot \varphi(m)=\varphi(m)^{\varphi(x)+1} .
$$

Since $N=\varphi(M)$ is a faithful $R$-module, we must have $\bar{\varphi}(x+1)=\bar{\varphi}(x)+1$. A straightforward calculation shows that $\bar{\varphi}(x)=x$ or $\bar{\varphi}(x)=x^{-1}$ so that the number of suitable automorphisms $\bar{\varphi}$ is finite. Since $\bar{\varphi}(x+1)=\bar{\varphi}(x)+1, \bar{\varphi}$ extends to an automorphism of $R$. Clearly $\varphi$ restricted to $M$ gives an $R$-module isomorphism from $M$ to $\bar{\varphi}_{N}$. Since the number of nonisomorphic modules $M$ is infinite and the number of suitable automorphisms $\bar{\varphi}$ is finite, the groups $G_{M}$ must lie in infinitely many isomorphism classes as required.

Now let $S=2\left[x, x^{-1},(x+1)^{-1}\right]$, a subring of $R$. As an $S$-module, 
$R$ is free. If $M$ is a projective $R$-module, it is a direct summand of a free $R$-module. Since $R$ is free over $S, M$ considered as an $S$-module is a direct summand of a free $S$-module and therefore is a projective $S$-module. Since all projective $S$-modules are free $([1, p .210])$, all of the $R$-modules used above are isomorphic, as $S$-modules, to $R$. Let $C$ be the subgroup of the group of units of $S$ generated by $x$ and $(x+1)$, and form the groups $H_{M}=M \rtimes C$. All the groups $H_{M}$ are isomorphic to $R \rtimes C=B$. For each module $M, G_{M}=M \rtimes A=H_{M} \rtimes I \cong B \rtimes I$ for a suitably chosen action of $I$ on $B$ via the isomorphism with ${ }_{M}$. Thus we have shown:

PROPOSITION. There are infinitely many nonisomorphic metabelian split extensions of the metabelian group $B$ by the finite abelian group II with the same finite quotients.

Let $x_{1}, \ldots, x_{m}$ be the elements of $\pi$. Then $\left\{x_{1}, \ldots, x_{m}\right\}$ is a basis of $R$ over $S$. Consider the group $G$ with presentation $\begin{aligned} &\left\langle t, s, y_{1}, \ldots, y_{m}\right|[t, s]=1, y_{i}^{s}=y_{i} y_{i}^{t},\left[y_{i}, y_{j}\right]=\left[y_{i}, y_{j}^{t}\right]=1, \\ &1 \leq i, j \leq m\rangle .\end{aligned}$

$G$ is clearly finitely presented and $G$ may be shown to be metabelian as in [3]. The map $t \rightarrow x, s \rightarrow(x+1), y_{i} \rightarrow x_{i}$ gives a homomorphism of $G$ onto $B=R \times C$. Since metabelian groups satisfy the maximal condition on normal subgroups [6], $B$ must be finitely presented. Since each $G_{M}$ is a finite extension of the finitely presented group $B$, we have proved:

PROPOSITION. Each of the groups $G_{M}$ is finitely presented.

\section{References}

[1] Hyman Bass, Algebraic K-theory (Benjamin, New York, Amsterdam, 1968).

[2] Hyman Bass and M. Pavaman Murthy, "Grothendieck groups and Picard groups of abelian group rings", Ann. of Math. (2) 86 (1967), 16-73. 
[3] Gilbert Baumslag, "A finitely presented metabelian group with a free abelian derived group of infinite rank", Proc. Amer. Math. Soc. 35 (1972), 61-62.

[4] Robert C. Brigham, "On the isomorphism problem for just-infinite groups", Comm. Pure App I. Math. 24 (1971), 789-796.

[5] Joan Landman Dyer, "On the isomorphism problem for polycyclic groups", Math. Z. 112 (1969), 145-153.

[6] P. Hall, "Finiteness conditions for soluble groups", Proc. London Math. Soc. (3) 4 (1954), 419-436.

[7] P. Hall, "On the finiteness of certain soluble groups", Proc. London Math. Soc. (3) 9 (1959), 595-622.

[8] M. Pavaman Murthy and Claudio Pedrini, " $K_{0}$ and $K_{1}$ of polynomial rings", Algebraic K-theory II, 109-121 (Proc. Conf. Seattle Research Center, Battelle Memorial Institute, 1972. Lecture Notes in Mathematics, 342. Springer-Verlag, Berlin, Heidelberg, New York, 1973).

[9] Paul Frederick Pickel, "On the isomorphism problem for finitely generated torsion free nilpotent groups", (PhD thesis, Rice University, Houston, Texas, 1970).

[10] P.F. Pickel, "Nilpotent-by-finite groups with isomorphic finite quotients", Trans. Amer. Math. Soc. 183 (1973), 313-325.

[11] В.Н. Ремесленнинов [V.N. Remeslennikov], "Сопряненность подгрупп в ннльпотентных группах" [Conjugacy of subgroups in nilpotent groups", Algebra i Logika 6 (1967), No. 2, 61-76.

[12] V.N. Remeslennikov, "Groups that are residually finite with respect to conjugacy", Siberian Math. J. 12 (1971), 783-792.

[13] Oscar Zariski and Pierre Samuel, Commutative algebra, Volume I (Van Nostrand, Princèton, New Jersey; Toronto; New York; London; 1958).

Department of Mathematics,

Polytechnic Institute of New York, Brooklyn, New York, USA. 